\title{
Raman-Induced Interactions in a Single-Component Fermi Gas Near an $s$-Wave Feshbach Resonance
}

\author{
R. A. Williams, M. C. Beeler, L. J. LeBlanc, K. Jiménez-García, and I. B. Spielman \\ Joint Quantum Institute, National Institute of Standards and Technology and University of Maryland, \\ Gaithersburg, Maryland 20899, USA
}

(Received 9 June 2013; published 26 August 2013)

\begin{abstract}
Ultracold gases of interacting spin-orbit-coupled fermions are predicted to display exotic phenomena such as topological superfluidity and its associated Majorana fermions. Here, we experimentally demonstrate a route to strongly interacting single-component atomic Fermi gases by combining an $s$-wave Feshbach resonance (giving strong interactions) and spin-orbit coupling (creating an effective $p$-wave channel). We identify the Feshbach resonance by its associated atomic loss feature and show that, in agreement with our single-channel scattering model, this feature is preserved and shifted as a function of the spin-orbit-coupling parameters.
\end{abstract}

DOI: 10.1103/PhysRevLett.111.095301

Feshbach resonances are characterized by singularities in two-body scattering, where a bound state becomes energetically degenerate with the two-body continuum. For ultracold atoms these resonances are generally tuned with external magnetic fields that Zeeman-shift the energy difference between the bound (molecular) state and the twoatom continuum. In two-component fermionic systems, $s$-wave Feshbach resonances enabled the experimental realization of the crossover from BCS pairing of fermions to the Bose-Einstein condensation (BEC) of bound molecules [1-4]. Exploring the same regime with spin-polarized Fermi gases will allow a transition to a new kind of superfluid - a topological superfluid - with stable Majorana fermions at system edges and at internal defects $[5,6]$. Owing to fermionic statistics, $s$-wave Feshbach resonances can only affect states of differing spin and therefore are absent in spin-polarized, single-component, Fermi gases. $p$-wave Feshbach resonances do allow this coupling, but have a large associated loss which limits their utility $[7,8]$.

In contrast, it was recently shown that effective $p$-wave interactions arise from $s$-wave interactions when combined with spin-orbit coupling [5,9]. A two-dimensional Fermi system with spin-orbit coupling is expected to exhibit chiral $p$-wave superfluidity with associated Majorana modes in vortices [5,10-12]. Similarly, trapped onedimensional spin-orbit-coupled systems are predicted to support Majorana fermions at their boundary [13-15]. A large body of theoretical work focuses on the essential two-body physics of a Feshbach resonance in the presence of spin-orbit coupling and the resulting modification of the BEC-BCS crossover [16-23]. To date, spin-orbit coupling

Published by the American Physical Society under the terms of the Creative Commons Attribution 3.0 License. Further distribution of this work must maintain attribution to the author(s) and the published article's title, journal citation, and DOI.
PACS numbers: 67.85.Lm, 03.75.Ss, 05.30.Fk, 34.50.-s

has been experimentally realized in both Bose gases [24-26] and Fermi gases [27,28]. In the latter case, radio frequency spectroscopy near a Feshbach resonance has been reported [29].

In this Letter, we experimentally study spin-orbitcoupled ${ }^{40} \mathrm{~K}$ atoms near a Feshbach resonance. Strong losses in atom number have been associated with Feshbach resonances in ultracold atoms since their initial observation [30]. We observe that the magnetic field value at which atom loss is maximum changes as a function of the strength and detuning of the Raman dressing which generates the spin-orbit coupling. We show that this change results from the energetically shifted Raman-dressed continuum coming into resonance with a bound molecular state. This effect of the Feshbach resonance shifting with the spin-orbit-coupling parameters has been predicted for the case of Rashba spin-orbit coupling [16,20,31-35], while more recent theoretical work has considered the equal mixture of Rashba and Dresselhaus spin-orbit coupling considered here [36,37].

We generate spin-orbit coupling in a Fermi gas by dressing the $\left|f=9 / 2, m_{F}=-7 / 2\right\rangle \equiv|\uparrow\rangle$ and $\mid f=9 / 2$, $\left.m_{F}=-9 / 2\right\rangle \equiv|\downarrow\rangle$ hyperfine spin states of ${ }^{40} \mathrm{~K}$ 's electronic ground state with a pair of counterpropagating Raman laser beams; see Fig. 1. These spin states exhibit a broad Feshbach resonance at $202.1 \mathrm{G}(1 \mathrm{G}=100 \mu \mathrm{T})$, with width $\simeq 7 \mathrm{G}[1,38]$. The Raman beams couple atoms in the $\left|\uparrow, k=q+k_{R}\right\rangle$ and $\left|\downarrow, k=q-k_{R}\right\rangle$ states [39] labeled by the quasimomentum $q$ and differing in linear momentum by $2 \hbar k_{R}$, where $\hbar k_{R}=2 \pi \hbar / \lambda$ is the recoil momentum and $\lambda=768.86 \mathrm{~nm}$ is the Raman laser's wavelength. The laser-dressed eigenstates $| \pm, q\rangle$ have a new single-particle dispersion relation consisting of two bands, shown in Fig. 1(b), and are quasimomentum-dependent superpositions of $|\uparrow\rangle$ and $|\downarrow\rangle$. The natural energy scale in the system is the recoil energy $E_{R}=\hbar \omega_{R}=\hbar^{2} k_{R}^{2} / 2 m$, with $\omega_{R}=2 \pi \times 8.445 \mathrm{kHz}$. The $\lambda=768.86 \mathrm{~nm}$ wavelength of 
(a) Level diagram

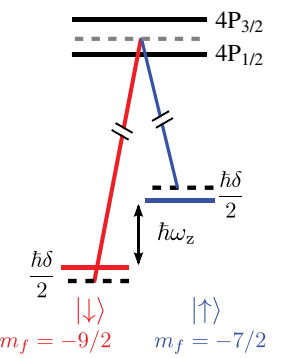

(b) Single-particle dispersion

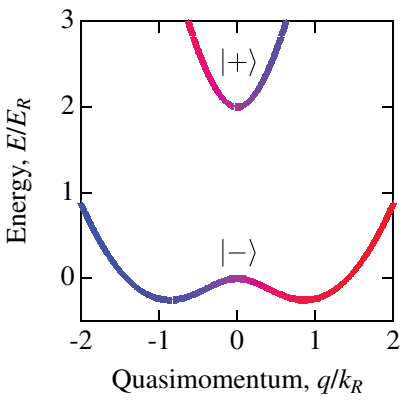

(c) Laser geometry

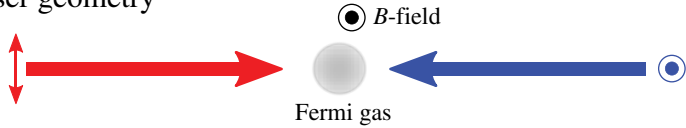

(d) Feshbach schematic

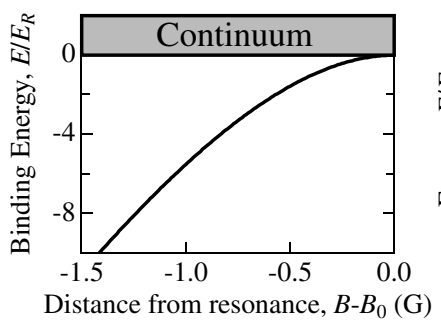

(e) Two-body energies

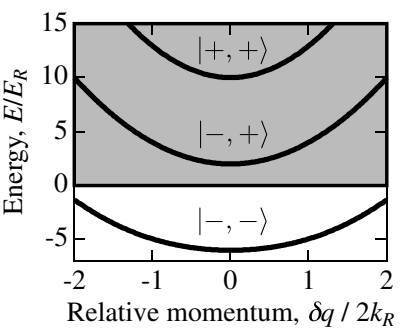

(f) Background resonance shift

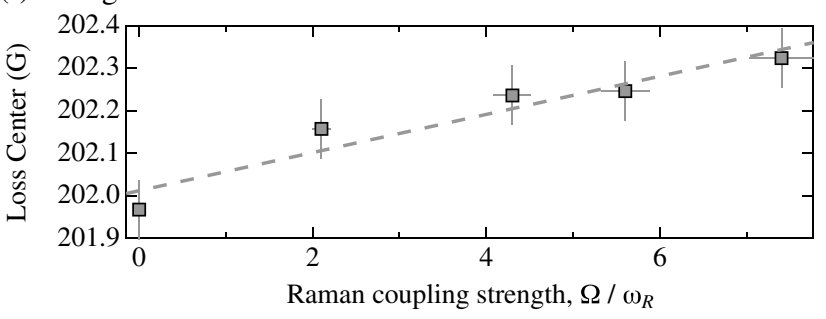

FIG. 1 (color online). (a),(c) Counterpropagating Raman beams couple two hyperfine spin states in ${ }^{40} \mathrm{~K}$ 's electronic ground state. (b) Energy bands for $\hbar \Omega=2 E_{R}$ and $\delta=0$. The color indicates the relative amplitude of the bare spin states in the dressed superposition. (d) Binding energy near the ${ }^{40} \mathrm{~K}$ Feshbach resonance. (e) Two-body energies showing the lowest band shift below the bare continuum for center-of-mass momentum $q_{\text {c.m. }}=0$ and relative momentum $q_{1}-q_{2}=2 \delta q$ for $\hbar \Omega=8 E_{R}$ and $\delta=0$. The upper and lower Raman-dressed bands are denoted using $|+\rangle$ and $|-\rangle$, respectively. (f) Center of loss feature versus Raman coupling strength $\Omega$ with a large two-photon detuning $\left(\hbar \delta=36 E_{R}\right)$ illuminating an incoherent spin mixture.

light providing the two-photon coupling is close to the zero-ac Stark shift magic wavelength such that the Raman beams contribute negligibly to the trapping potential. The single-particle spin-orbit-coupled system is completely characterized by the Raman coupling strength $\Omega$ and the two-photon detuning $\delta=\omega_{Z}-\Delta \omega_{L}$, where $\Delta \omega_{L}$ is the angular frequency difference between the Raman beams and $\hbar \omega_{Z}$ is the Zeeman splitting between the $|\uparrow\rangle$ and $|\downarrow\rangle$ states. Note that the minimum of the lower Raman-dressed band is shifted downwards in energy as $\Omega$ increases; when $\Omega \gg 4 E_{R}$, this shift is $\approx \sqrt{\left(\Omega^{2}+\delta^{2}\right)} / 2$.

Before studying the effect of Raman coupling on the Feshbach resonance, we carry out a control experiment to study the effect of the laser light independent of Raman dressing. We create a spin mixture of atoms in $|\uparrow\rangle$ and $|\downarrow\rangle$, illuminate the atoms with light that is far detuned from Raman resonance $\left(\hbar \delta=36 E_{R}\right)$, and measure atom loss near the Feshbach resonance. Surprisingly, this offresonant light shifts the loss feature to higher magnetic fields as shown in Fig. 1(f). This shift also exists for illumination by a single laser beam, confirming it is independent of the Raman-dressed physics of concern here. We attribute this shift (effectively $45 \mathrm{mG} / E_{R}$ ) to a differential ac-Stark shift between the open channel atoms and molecular states (most likely the closed channel component) involved in the Feshbach resonance. We find it exists over a range of wavelengths (while photoassocation-type effects associated with optical Feshbach resonances usually occur at a specific wavelength $[40,41]$, a recent experiment in ${ }^{40} \mathrm{~K}$ reported significant optical shifts of the $202.1 \mathrm{G}$ resonance from bound-bound transitions [42] over a range of wavelengths).

At the simplest level, a Feshbach resonance occurs when two requirements are satisfied: (i) a pair of colliding atoms in the continuum must be degenerate with a molecular state, and (ii) the matrix element coupling the atomic and molecular states must be nonzero. That the Raman laser coupling induces the Feshbach resonance and shifts its location relies on both of these facts: (i) the lower of the Raman-dressed bands is shifted downwards in energy, bringing potentially scattering atoms into resonance with an otherwise bound molecule, and (ii) even within the same band, colliding atoms-each of which is a momentumdependent spin superposition-couple to the molecular state by the usual $s$-wave coupling. The finite momentum width of the Fermi gas ensures atoms exist in different superpositions of the bare spin states; while these fermions share the same "dressed" band index, they are microscopically nonidentical and can interact with each other.

Sufficiently near a Feshbach resonance, the scattering takes on a universal form and only the last bound state approaching the continuum is relevant. In this limit, the bound state's energy $E_{B}=\hbar^{2} / m a^{2}$ is simply related to the scattering length $a$ and the atomic mass $m$ [43]. Figure 1(d) shows the energy of the last bound state computed for ${ }^{40} \mathrm{~K}$ in the universal regime. In comparison, Fig. 1(e) shows the two-body Raman-dressed state energies, highlighting that Raman-dressed atoms in the lowest band lie below the initial continuum. We therefore anticipate a scattering resonance when the Raman-dressed continuum is degenerate with the initial molecular state.

The experiments begin with a spin-polarized gas of around $1.5 \times 10^{5}{ }^{40} \mathrm{~K}$ atoms in the $|\downarrow\rangle$ state at a temperature of $T / T_{F}=0.4$. This degenerate Fermi gas is trapped 
in a crossed optical dipole trap with frequencies $\left\{\omega_{x}, \omega_{y}, \omega_{z}\right\} / 2 \pi=\{39,42,134\} \mathrm{Hz}$.

A drawback of the current generation of experiments investigating spin-orbit coupling in alkali atoms is the spontaneous emission from off-resonantly excited atoms, leading to heating and loss. Here this problem is mitigated by using a relatively fast (in the sense of not being limited by the trap frequency time scale) procedure to load fermions into the lowest Raman-dressed band. We begin with a noninteracting spin-polarized Fermi gas in the $|\downarrow\rangle$ state at the desired magnetic field $B$, setting the distance from the Feshbach resonance. We then ramp the Raman beams to their final power in $2 \mathrm{~ms}$, slow compared to interband energy scales but fast compared to the trap frequencies. This loads the Fermi gas into the lowest Raman-dressed band with an average quasimomentum $q=k_{R}$, and thus a nonzero group velocity dependent on $\Omega$ and $\delta$. We confirmed that no atoms are loaded into the higher energy Raman band, ruling out the possibility of collisions between $|-\rangle$ and $|+\rangle$ atoms.

Nearly all the experiments described in this Letter share this procedure, after which the Raman-dressed fermions are held in the optical dipole trap for a variable time. As the atoms move in the trap and change their quasimomentum, their internal state evolves adiabatically; for example, upon reaching $q=0$ an atom is an equally weighted superposition of $\left|\uparrow, q+k_{R}\right\rangle$ and $\left|\downarrow, q-k_{R}\right\rangle$. We consider two scenarios: (i) for $\Omega>4 E_{R}$ we allow the Raman-dressed cloud to undergo a quarter oscillation in the trap, at which point its center-of-mass momentum is zero, (ii) for $\Omega<4 E_{R}$ the cloud initially sits in one side of the double-well dispersion and has negligible initial group velocity.

We first investigate how the atomic loss feature of a spinorbit-coupled Fermi gas near a Feshbach resonance depends on parameters $\delta$ and $\Omega$. Figure 2(a) shows the raw data from such experiments, in which the detuning $\delta$ is varied at fixed $\Omega$. The different colored data sets represent different values of $\delta$. The detuning is controlled at a given magnetic field (setting $\omega_{Z}$ ) by changing the frequency difference $\Delta \omega_{L}$ between the Raman beams using acousto-optic modulators.

In this experiment, we keep the Raman coupling strength constant at $\hbar \Omega=4.7 E_{R}$. The initially moving Raman-dressed atoms are held in the dipole trap for $14 \mathrm{~ms}$. For $\hbar \Omega=4.7 E_{R}, \delta=0$, this is the time the ${ }^{40} \mathrm{~K}$ cloud takes to come to rest in the trap. This hold time is longer than a quarter period in the bare trap due to the modified dispersion relation of the dressed fermions. After this hold time, the dipole trap is suddenly turned off $(<1 \mu \mathrm{s})$. At the beginning of time-of-flight (TOF) the Raman-dressed atoms are mapped back to the bare spin state $|\downarrow\rangle$ by sweeping the detuning by an additional $\hbar \delta=$ $20 E_{R}$ in $1 \mathrm{~ms}$ and then turning off the Raman lasers in $1 \mathrm{~ms}$. We absorption image the ${ }^{40} \mathrm{~K}$ cloud after a total TOF of $6.3 \mathrm{~ms}$ and count the remaining atoms.

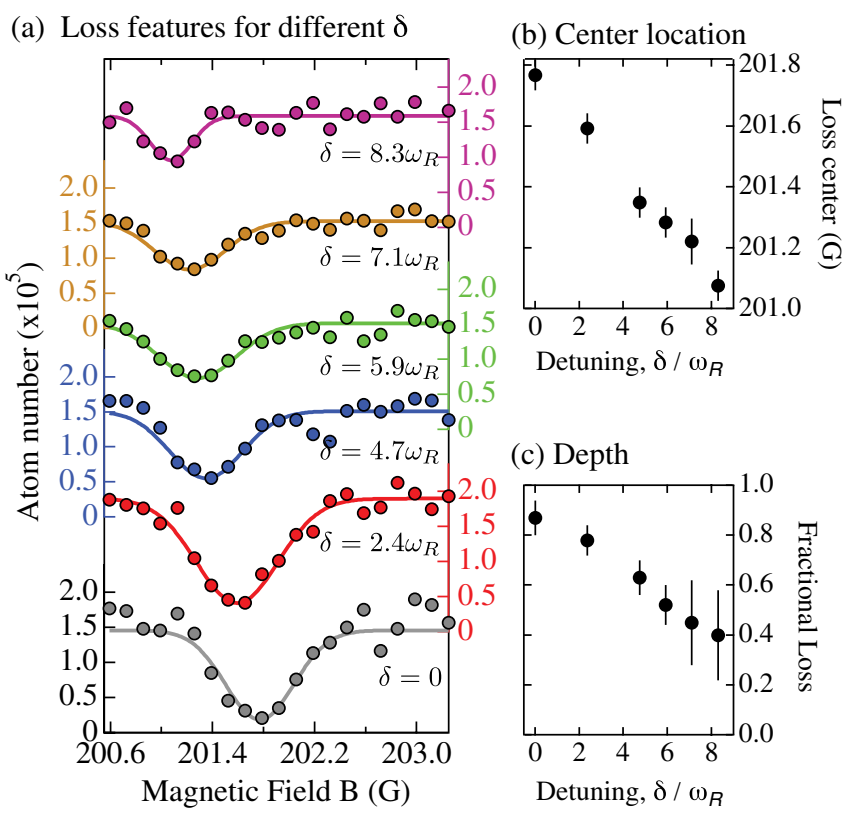

FIG. 2 (color online). (a) Atom number as a function of magnetic field for $\hbar \Omega=4.7 E_{R}$ and detunings $\delta$ : gray, $\hbar \delta=0$; red, $\hbar \delta=2.4 E_{R}$; blue, $\hbar \delta=4.7 E_{R}$; green, $\hbar \delta=5.9 E_{R}$; orange, $\hbar \delta=7.1 E_{R} ;$ magenta, $\hbar \delta=8.3 E_{R}$. The solid curves are Gaussian fits to the data. (b) Center of loss feature as a function of $\delta$. (c) Fraction of atoms lost as a function of $\delta$. Uncertainty bars represent 1 standard deviation.

We observe that with increasing $\delta$ (i) the absolute magnetic field value at which maximum loss occurs shifts to smaller values [Fig. 2(b), raw data, uncorrected for the off-resonant effect of Fig. 1(f)], (ii) the total atom loss in a fixed time decreases [Fig. 2(c)]. The first observation can be explained by the downward shifted Raman-dressed continuum coming into resonance with a bound molecular state. The second observation results from the increasing spin polarization of the dressed state (and hence reduced coupling to the molecular state) as $\delta$ increases.

To theoretically account for these results, we extend a single-channel scattering model to include Raman coupling between the two spin states. We identify the shifted bound states from poles in the Green function. Figure 3 shows the calculated energy of the last molecular bound state (indicated by the color scale; the white regions signify that no molecule is present) as a function of $\delta$ or $\Omega$ for a center-of-mass momentum $\hbar q_{\text {c.m. }}$. The red curve denotes the prediction of a simple model equating $E_{B}$ to the energy of a pair of atoms at the energy minimum of the lowest Raman-dressed band.

In a second measurement, we investigate loss near the Feshbach resonance as a function of the Raman coupling strength $\Omega$, with the detuning $\delta=0$. As previously, we load the ${ }^{40} \mathrm{~K}$ atoms into the lowest energy Raman-dressed band and hold the atoms in the optical dipole trap such that the Fermi gas only performs a single quarter oscillation. The hold times are between 10 and $15 \mathrm{~ms}$ over the range of 

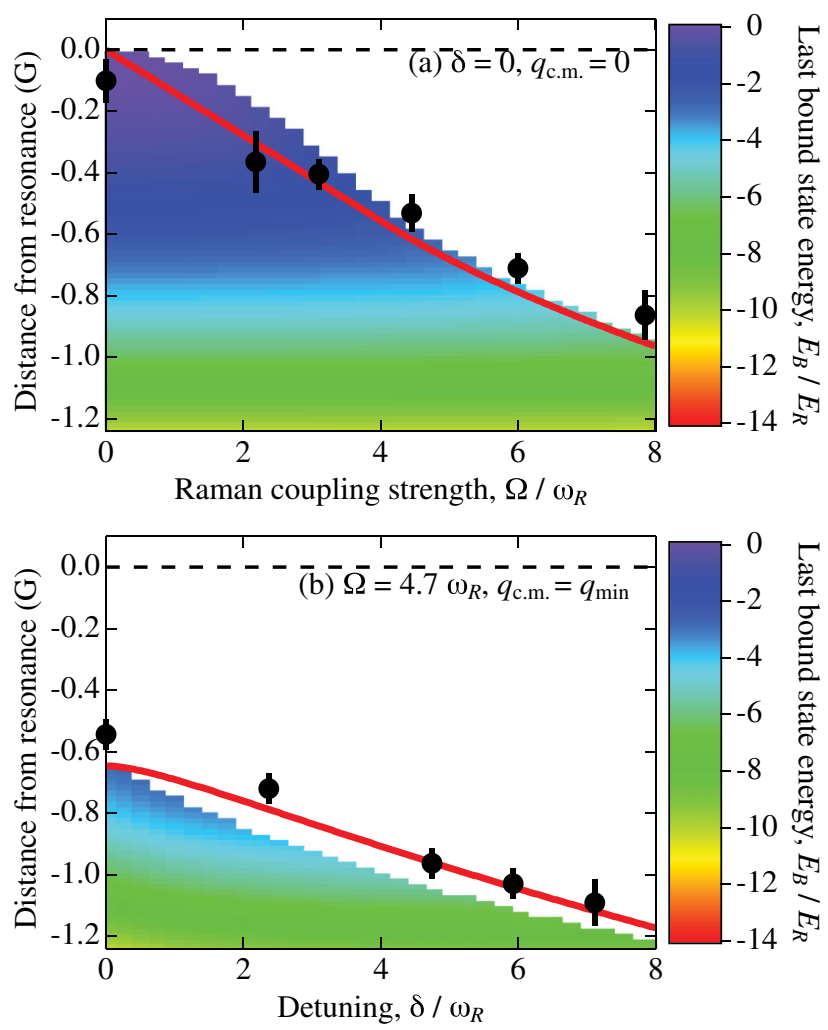

FIG. 3 (color online). Predicted energy of the last bound molecular state as a function of (a) Raman coupling strength $\Omega$ for $\delta=0$ and (b) $\delta$ for $\hbar \Omega=4.7 E_{R}$. The color scale indicates the energy of the last bound state with center-of-mass momentum $h q_{\text {c.m. }}$, and the white region denotes where the bound state has entered the continuum and becomes a scattering state. The red curve denotes the prediction of the simple model discussed in the text. Experimental data, with the background shift of Fig. 1(f) subtracted, is shown by the black circles.

coupling strength we use. After this hold time, we determine the remaining number of atoms and map out the atomic loss signal as a function of magnetic field.

We compare the results of these experiments to theory in Fig. 3, having now subtracted the intensity-dependent background shift of Fig. 1(f). The black symbols in Fig. 3(a) plot the monotonically decreasing location of the loss feature as a function of Raman coupling $\Omega$, showing semiquantative agreement with theory. We determine the position of maximal atomic loss in the absence of spinorbit coupling $(\Omega=0)$ using a spin mixture rather than an initially spin-polarized Fermi gas. Likewise, Fig. 3(b) depicts the loss feature moving to lower magnetic fields as a function of increasing detuning $\delta$, again in accord with theory. In both of these cases, we load the Fermi gas into only the lowest Raman dressed state, where it is effectively a single-component system. The continued effect of the Feshbach resonance in this polarized gas is indicative of the effective $p$-wave interactions between these laserdressed fermions-just as laser-dressed bosons acquire effective $d$-wave interactions [9].

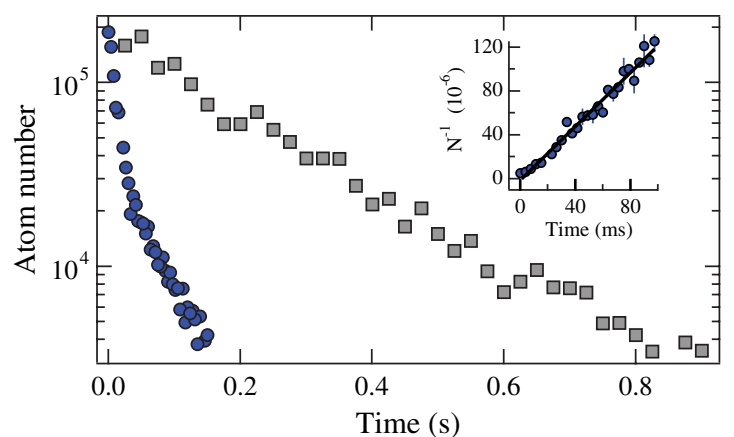

FIG. 4 (color online). Atom number versus hold time in an optical dipole trap for different scenarios: (i) gray squares, $B=201.9 \mathrm{G}, \quad \hbar \Omega=2.1 E_{R}, \quad \hbar \delta=33 E_{R}, \quad$ (ii) blue circles, $B=201.9 \mathrm{G}, \hbar \Omega=2.1 E_{R}, \hbar \delta=0$. Inset: The data of (ii) plotted as $1 / N$. The linear time dependence of $1 / N$ is consistent with a two-body decay process.

Finally, we consider the rates associated with different loss mechanisms in the experiment. Figure 4 shows the number of atoms as a function of hold time in the optical dipole trap for two different scenarios. The gray data display the decay of a spin-polarized gas illuminated with off-resonant Raman beams $\left(\Omega=2.1 E_{R}, \delta=33 E_{R}\right)$. In this case the atom loss is only due to the scattering of photons from the Raman lasers, giving a single-body decay with $1 / e$ time of $180 \mathrm{~ms}$ (the loss rate due to collisions with background atoms is negligible on the time scale of this experiment). This comparatively slow rate implies that our $\approx 14 \mathrm{~ms}$ procedure for measuring loss is unaffected by spontaneous emission.

In contrast, the blue circles show atom numbers for the on-resonant Raman beam case $\left(\Omega=2.1 E_{R}, \delta=0\right)$. We load the atoms into the lowest dressed band as described previously, at a magnetic field $B=201.9 \mathrm{G}$ (close to the field for maximum loss rate for this Raman coupling). The loss curve agrees well with that expected for a two-body loss process, as highlighted by the inset of Fig. 4(b), showing that the inverse of the atom number is linear in time.

Loss near a Feshbach resonance occurs in a two-step process whereby three-body collisions result in the formation of shallow dimers (which are not lost from the trap), followed by two-body collisions that allow the shallow dimers to decay to much more deeply bound molecules [44]. The large kinetic energy released in this decay results in loss from the trap. It is possible that the demapping (back to a single spin state) step in the TOF (for which $\delta$ is increased by ramping $B$ ) dissociates the shallow dimers, allowing them to be imaged and counted. This would make the loss rate resemble a two-body process, although twobody decay has previously been observed for fermions near a Feshbach resonance [45].

In summary, we explored atomic loss in a spin-orbitcoupled Fermi gas near a Feshbach resonance. At sufficiently low temperatures this system in 1D (and possibly 
even 2D and 3D [46]) is expected to allow topological superfluidity and support Majorana fermions.

We benefited greatly from conversations with C. A. R. Sa de Melo, V. B. Shenoy, and E. Tiesinga. This work was partially supported by the ARO with funding from the DARPA OLE program and the Atomtronics-MURI, and by the NSF through the PFC at JQI. L. J. L. thanks NSERC and M.C.B. thanks the NIST-ARRA program.

[1] C. A. Regal, M. Greiner, and D. S. Jin, Phys. Rev. Lett. 92, 040403 (2004).

[2] M. Bartenstein, A. Altmeyer, S. Riedl, S. Jochim, C. Chin, J. H. Denschlag, and R. Grimm, Phys. Rev. Lett. 92, 120401 (2004).

[3] M.W. Zwierlein, C.A. Stan, C.H. Schunck, S.M.F. Raupach, A.J. Kerman, and W. Ketterle, Phys. Rev. Lett. 92, 120403 (2004).

[4] T. Bourdel, L. Khaykovich, J. Cubizolles, J. Zhang, F. Chevy, M. Teichmann, L. Tarruell, S. J. J. M. F. Kokkelmans, and C. Salomon, Phys. Rev. Lett. 93, 050401 (2004).

[5] C. Zhang, S. Tewari, R. M. Lutchyn, and S. D. Sarma, Phys. Rev. Lett. 101, 160401 (2008).

[6] D. A. Ivanov, Phys. Rev. Lett. 86, 268 (2001).

[7] C. A. Regal, C. Ticknor, J. L. Bohn, and D. S. Jin, Phys. Rev. Lett. 90, 053201 (2003).

[8] J. Levinsen, N. R. Cooper, and V. Gurarie, Phys. Rev. A 78, 063616 (2008).

[9] R. A. Williams, L. J. LeBlanc, K. Jiménez-García, M. C. Beeler, A. R. Perry, W. D. Phillips, and I. B. Spielman, Science 335, 314 (2012).

[10] M. Sato, Y. Takahashi, and S. Fujimoto, Phys. Rev. Lett. 103, 020401 (2009).

[11] S.-L. Zhu, L.-B. Shao, Z. D. Wang, and L.-M. Duan, Phys. Rev. Lett. 106, 100404 (2011).

[12] M. Gong, G. Chen, S. Jia, and C. Zhang, Phys. Rev. Lett. 109, 105302 (2012).

[13] L. Jiang, T. Kitagawa, J. Alicea, A. R. Akhmerov, D. Pekker, G. Refael, J. I. Cirac, E. Demler, M. D. Lukin, and P. Zoller, Phys. Rev. Lett. 106, 220402 (2011).

[14] X.-J. Liu, L. Jiang, H. Pu, and H. Hu, Phys. Rev. A 85, 021603 (2012).

[15] R. Wei and E. J. Mueller, Phys. Rev. A 86, 063604 (2012).

[16] J.P. Vyasanakere and V.B. Shenoy, Phys. Rev. B 83, 094515 (2011).

[17] L. Jiang, X.-J. Liu, H. Hu, and H. Pu, Phys. Rev. A 84, 063618 (2011).

[18] J. Zhou, W. Zhang, and W. Yi, Phys. Rev. A 84, 063603 (2011).

[19] T. Ozawa and G. Baym, Phys. Rev. A 84, 043622 (2011).

[20] Z.-Q. Yu and H. Zhai, Phys. Rev. Lett. 107, 195305 (2011).
[21] L. Han and C. A. R. Sá de Melo, Phys. Rev. A 85, 011606 (2012).

[22] L. He and X.-G. Huang, Phys. Rev. Lett. 108, 145302 (2012).

[23] J.-X. Cui, X.-J. Liu, G. L. Long, and H. Hu, Phys. Rev. A 86, 053628 (2012).

[24] Y.-J. Lin, K. Jiménez-García, and I. B. Spielman, Nature (London) 471, 83 (2011).

[25] J.-Y. Zhang, S.-C. Ji, Z. Chen, L. Zhang, Z.-D. Du, B. Yan, G.-S. Pan, B. Zhao, Y.-J. Deng, H. Zhai, S. Chen, and J.-W. Pan, Phys. Rev. Lett. 109, 115301 (2012).

[26] C. Qu, C. Hamner, M. Gong, C. Zhang, and P. Engels, arXiv:1301.0658.

[27] P. Wang, Z.-Q. Yu, Z. Fu, J. Miao, L. Huang, S. Chai, H. Zhai, and J. Zhang, Phys. Rev. Lett. 109, 095301 (2012).

[28] L. W. Cheuk, A. T. Sommer, Z. Hadzibabic, T. Yefsah, W. S. Bakr, and M. W. Zwierlein, Phys. Rev. Lett. 109, 095302 (2012).

[29] Z. Fu, L. Huang, Z. Meng, P. Wang, X.-J. Liu, H. Pu, H. Hu, and J. Zhang, Phys. Rev. A 87, 053619 (2013).

[30] J. Stenger, S. Inouye, M. R. Andrews, H.-J. Miesner, D. M. Stamper-Kurn, and W. Ketterle, Phys. Rev. Lett. 82, 2422 (1999).

[31] A. V. Chaplik and L. I. Magarill, Phys. Rev. Lett. 96, 126402 (2006).

[32] E. Cappelluti, C. Grimaldi, and F. Marsiglio, Phys. Rev. Lett. 98, 167002 (2007).

[33] M. Gong, S. Tewari, and C. Zhang, Phys. Rev. Lett. 107, 195303 (2011).

[34] V. B. Shenoy, arXiv:1212.2858.

[35] L. Zhang, Y. Deng, and P. Zhang, Phys. Rev. A 87, 053626 (2013).

[36] V. B. Shenoy (unpublished).

[37] D. M. Kurkcuoglu and C.A.R. Sá de Melo, arXiv:1306.1964.

[38] U. Schneider, L. Hackermuller, J. P. Ronzheimer, S. Will, S. Braun, T. Best, I. Bloch, E. Demler, S. Mandt, D. Rasch, and A. Rosch, Nat. Phys. 8, 213 (2012).

[39] Y.-J. Lin, R. L. Compton, A. R. Perry, W. D. Phillips, J. V. Porto, and I. B. Spielman, Phys. Rev. Lett. 102, 130401 (2009).

[40] D. M. Bauer, M. Lettner, C. Vo, G. Rempe, and S. Dürr, Nat. Phys. 5, 339 (2009).

[41] D. M. Bauer, M. Lettner, C. Vo, G. Rempe, and S. Dürr, Phys. Rev. A 79, 062713 (2009).

[42] Z. Fu, P. Wang, L. Huang, Z. Meng, H. Hu, and J. Zhang, arXiv:1306.0395.

[43] C. Chin, R. Grimm, P. Julienne, and E. Tiesinga, Rev. Mod. Phys. 82, 1225 (2010).

[44] S. Zhang and T.-L. Ho, New J. Phys. 13, 055003 (2011).

[45] K. Dieckmann, C. A. Stan, S. Gupta, Z. Hadzibabic, C. H. Schunck, and W. Ketterle, Phys. Rev. Lett. 89, 203201 (2002).

[46] K. Seo, L. Han, and C. A. R. Sá de Melo, Phys. Rev. Lett. 109, 105303 (2012). 